\title{
"Es un poco cobarde mi tipo de escritura": formas de citación y negociaciones identitarias de doctorantes en Educación
}

\author{
"It's a bit cowardly my kind of writing": citation forms and \\ identity negotiations of doctoral students in Education
}

\author{
Lina Calle-Arango' \\ Natalia Ávila Reyes ${ }^{2}$ \\ Alejandra Meneses ${ }^{3}$
}

\section{RESUMEN}

Este artículo presenta cuatro casos de estudio orientados por las preguntas de investigación: ¿cómo los estudiantes doctorales distribuyen sus formas de citación en una misma práctica letrada?, ¿qué cambios tienen estas distribuciones a lo largo de diferentes versiones del texto producidas durante un año y medio de formación doctoral? y ¿qué razones atribuyen a estas elecciones y preferencias de uso? Para responderlas, se analizó el estilo e integración de las citas en tres versiones por escritor, y se contrastó con entrevistas semiestructuradas. Este doble abordaje metodológico

1. Pontificia Universidad Católica de Chile, Santiago de Chile, Facultad de Educación. https://orcid.org/0000-0003-4362-3075. Email: linacallearango@gmail.com

2. Pontificia Universidad Católica de Chile, Santiago de Chile, Facultad de Educación. https://orcid.org/0000-0003-3528-6806.Email: naavila@uc.cl

3. Pontificia Universidad Católica de Chile, Santiago de Chile, Facultad de Educación, Departamento de Didáctica. https://orcid.org/0000-0002-6563-5117. Email: amenesea@ uc.cl 
contribuye a la tradición en crecimiento de estudios sobre escritura en posgrado interesados en las relaciones entre las elecciones discursivas del escritor y la construcción de su identidad académica.

Palabras clave: citación; escritura académica; identidad.

\begin{abstract}
This paper presents four case studies guided by the research questions: how do doctoral students distribute their citation forms in the same literary practice, how do these distributions change across different versions of the text produced during a year and a half of doctoral training, and what reasons do they attribute to these choices and preferences of use? To answer them, we analyzed the style and integration of citations in three versions per writer, and contrasted with semi-structured interviews. This combined methodological approach contributes to the growing tradition of postgraduate writing studies interested in the relationships between the writer's discursive choices and the construction of his or her academic identity.
\end{abstract}

Keywords: citation; academic writing; identity.

\title{
1. Introducción
}

Aunque la investigación sobre escritura en estudiantes de posgrado ha incrementado recientemente, aún es menor que la realizada en pregrado (Chois \& Jaramillo, 2016; Sala-Bubaré \& Castelló, 2018; Simpson, 2016) y continúa siendo incipiente en Latinoamérica (CalleArango \& Ávila Reyes, 2020). La creciente atención a la escritura en posgrado responde, en parte, al aumento acelerado de programas doctorales durante las últimas décadas (Dávila, 2012; OECD, 2019; UNESCO \& IESLAC, 2007; White \& Grinnell, 2011).

Muchas de estas investigaciones han indagado sobre aspectos relacionados con la identidad de estudiantes de posgrado desde marcos teóricos diversos, como dan cuenta revisiones bibliográficas recientes (ver Castelló et al., de 2020; Choi et al., 2021; Inouye \& McAlpine, 2019). Un primer grupo de investigaciones indaga acerca del desarrollo 
de estos escritores a partir de recursos discursivos que extienden los trabajos seminales de Hyland (1999) y Swales (1990). Entre los focos más recurrentes están la identificación de marcas metadiscursivas (p.e. Castro \& Sánchez, 2016; McCambridge, 2019) y el análisis de estructuras o movidas retóricas de tesis en una disciplina (p.e. Difabio De Anglat \& Álvarez, 2019), o bien la comparación entre disciplinas (p.e. Martínez, 2015; Solar, 2013) o lenguas (p.e. Araújo, 2006). Estos estudios se han enfocado mayoritariamente en los comportamientos discursivos de los escritores, partiendo del análisis de los productos escritos para interpretar cómo los estudiantes se desarrollan. Entre ellos, solo el trabajo de McCambridge (2019) ofrece un vínculo entre el uso de recursos específicos y la construcción identitaria.

Un segundo grupo de trabajos sobre escritura en posgrado estudia la intertextualidad (p.e. Badenhorst, 2019; Canata et al., 2017; Castro \& Sánchez, 2016; Chiware \& Becker, 2018; Salami \& Olatokun, 2018; Swart, 2019). Dado que la identidad discursiva es una construcción social, analizar la intertextualidad permite adentrarse en las formas retóricas en que se posiciona el escritor (Bazerman, 2004), pues mediante las citas que incorpora adhiere con determinadas líneas teóricas y epistemológicas, toma distancia de otras propuestas o incluso se opone. Muchas de estas investigaciones han ahondado en el análisis textual de comportamientos y patrones de citación. Algunas identifican los tipos de documentos (artículos académicos, libros, conferencias, etc.) más citados por los estudiantes de postgrado (p.e. Canata et al., 2017; Chiware \& Becker, 2018; Salami \& Olatokun, 2018; Swart, 2019), mientras otras contabilizan frecuencias y distinguen entre estilo - directo e indirecto-e integración (p.e. Castro \& Sánchez, 2016; Badenhorst, 2019; Meza Guzmán, 2013; Venegas et al., 2013) para explicar comportamientos de los escritores y su ajuste a convenciones discursivas determinadas.

Este último grupo de estudios sobre intertextualidad ofrece análisis contrastivos entre ciclos formativos de educación superior y comunidades disciplinares, como el trabajo de Venegas et al. (2013), que analizó el estilo y la integración de citas en tesis de pregrado y magíster en Lingüística y Filosofía. Los resultados mostraron que las mayores diferencias en el uso de citas se obtienen comparando nivel formativo más que disciplina. En maestría se usa más del doble de 
citas indirectas que en licenciatura, y los estilos preferidos por los estudiantes son las citas indirectas en ambos niveles educativos en Lingüística, y en Licenciatura en Filosofía. En el análisis de Meza Guzmán (2013) de 26 tesis de Lingüística distribuidas equitativamente entre licenciatura, magíster y doctorado, la cita indirecta también emergió como predilecta entre estudiantes de los tres niveles, aunque su uso va disminuyendo conforme se avanza en grado académico. Sobre la integración, hubo mayor inclinación por las citas no integrales en el nivel doctoral, contrario a los otros dos ciclos formativos. Un estudio similar analizó los tipos de citas en cuatro tesis doctorales en Filosofía de una universidad pública mexicana (Castro \& Sánchez, 2016) siguiendo la propuesta de Massi (2005). Los resultados mostraron predominancia de la cita integral con presencia en las tres secciones de los textos -introducción, aporte personal y conclusión-, y con mayor aparición en los apartados que requieren sustento de aportes personales, lo que sugiere el uso de este estilo como un patrón que se explica disciplinarmente. Por su parte, Badenhorst (2019) estudió la "flexibilidad intertextual" partiendo de los patrones de citación identificados en 23 borradores y 23 versiones finales de revisiones de literatura generadas en un curso seminal por estudiantes de maestría en Educación. Partiendo de los conteos de citación e identificación del estilo e integración de las citas, la autora construyó tres categorías de escritores - de alta, media y baja flexibilidad intertextual-, que corresponden a grados de avance en la habilidad para proporcionar perspectivas holísticas de las fuentes consultadas, parafrasear las citas y recontextualizar las ideas conforme a la línea argumental construida.

Los estudios sobre intertextualidad en postgrado hasta acá reportados han constituido un gran aporte para comprender las tendencias y prácticas de citación de escritores en formación. Sin embargo, se han enfocado en análisis de los textos por parte de los investigadores, sin incorporar otras fuentes de información para comprender lo que motiva sus elecciones discursivas. Tampoco indagan la construcción de la identidad de los escritores, más bien, esta se infiere de sus posicionamientos textuales. Asimismo, sus resultados tienden a sugerir un carácter monolítico de las convenciones en las comunidades discursivas (Ivanič, 1998), que limita implícitamente las posibilidades de elecciones discursivas de los estudiantes. 
Un enfoque menos explorado ha aportado una visión integradora de la citación y de las percepciones de los escritores sobre sus elecciones desde el análisis de los textos y de entrevistas. Esto ocurre en el trabajo de Shi et al. (2018), quienes entrevistan a escritores de posgrado de diferentes disciplinas para comprender sus percepciones y prácticas sobre la paráfrasis. Para ello, utilizaron como base la identificación de cláusulas de parafraseo en textos producidos por los entrevistados. Los participantes provenientes de disciplinas "duras" comentaron más que aquellos de "blandas" cómo incluían su propia interpretación al parafrasear, mientras que estos últimos tematizaron más frecuentemente cómo omitían información del texto original al construir la paráfrasis.

También Jomaa y Bidin (2019) analizaron cualitativamente citas (integración y estilo) de revisiones de literatura de 20 tesis en Lingüística Aplicada e Información Tecnológica escritas por doctorandos en una universidad malaya. El análisis se comparó con entrevistas basadas en textos (text-based interviews) a nueve de estos escritores para comprender sus percepciones sobre las formas de citación. Las entrevistas sugirieron que la decisión de uso de citas integrales o no integrales responde al tipo de fuente de información que se referencia y, asimismo, se relaciona con intenciones como destacar o enfatizar ideas que consideran relevantes, llamar la atención del lector e intensificar la credibilidad de la información proporcionada.

Además, algunas investigaciones que combinan el análisis de textos y entrevistas ahondan explícitamente en la relación entre la citación y el desarrollo de la identidad del escritor desde una perspectiva sociocultural. Estos avances han relevado las estrategias desplegadas por los investigadores en formación, tales como demostrar conocimiento de las autoridades reconocidas en el área de estudio o disimular poca familiaridad con un tema (Fazel \& Shi, 2015), recrear identidades que se ajusten a las expectativas del lector (Calle-Arango et al., 2021; Harwood \& Petrić, 2012), atender a los consejos de sus directores de tesis o emular a otros escritores (Fazel \& Shi, 2015), y tomar decisiones basadas en prácticas previas que los relacionan con sus identidades profesionales (Calle-Arango et al., 2021) o con sus identidades de escritores académicos aún nóveles (Qingrong \& Liming, 2019). 
El último conjunto de estudios apunta a comprender más integralmente una práctica discursiva compleja como es la intertextualidad académica, cuyo dominio trasciende el aprendizaje de convenciones e implica la movilización de recursos lingüísticos para construir una voz autoral autorizada. Al combinar análisis de textos e indagaciones sobre las motivaciones de los escritores, puede observarse el fenómeno en su complejidad, y comprender mejor las tensiones que experimentan los escritores en formación en la construcción de esa voz (Castelló, 2016; Fisher et al., 2020).

La presente investigación pretende aportar en esta dirección planteándose como objetivo determinar los cambios en las formas de citación de doctorandos en Educación durante la escritura iterada de una práctica letrada específica y su relación con las razones que ellos atribuyen a estas elecciones discursivas. Para conseguirlo, se formularon las siguientes preguntas: ¿cómo los estudiantes doctorales distribuyen sus formas de citación en una misma práctica letrada?, ¿qué cambios tienen estas distribuciones a lo largo de diferentes versiones del texto producidas durante un año y medio de formación doctoral? y ¿qué razones atribuyen los estudiantes a estas elecciones y preferencias de uso? Estas preguntas permiten comprender las tensiones que emergen al adquirir una nueva identidad como investigadores en Educación, entendiendo que las formas de citación constituyen indicios de estrategias para construir o negociar una identidad discursiva, y dialogan con las prácticas legitimadas y privilegiadas en las distintas comunidades discursivas. Para esto, se propuso un análisis de las formas de citación en la construcción sostenida de una práctica específica durante un año y medio, y se contrastó con entrevistas a sus escritores. Este artículo busca contribuir al campo de investigación de la escritura desde una visión sociocultural de la identidad de los escritores, que ha develado la importancia de desestimar la escritura como producto y comprenderla como una práctica social; y que, pese a los importantes avances en el conocimiento sobre las prácticas letradas en contextos de educación superior, ha indagado poco la formación de futuros investigadores, sus elecciones de uso y su relación con el complejo proceso de construcción de identidades académicas. 


\section{Marco teórico}

\section{Identidades discursivas}

Desde la perspectiva de las "Literacidades Académicas", la identidad es un constructo múltiple y socioculturalmente construido, que se cristaliza en un repertorio personal de recursos para actuar en situaciones de comunicación que los escritores construyen a partir de las afiliaciones a creencias y posibilidades de ser disponibles en los contextos culturales a los que van teniendo acceso (Burgess \& Ivanič, 2010; Ivanič, 1998). En este sentido, el sujeto puede identificarse simultáneamente con prácticas privilegiadas por distintas comunidades discursivas y elige, a veces inconscientemente, qué utilizar según el contexto. Así, la identidad está permeada por los discursos que el escritor adopta a lo largo de su vida, pero también por cómo los usa, interpreta y resignifica (Ivanič, 1998). Participar de nuevos contextos culturales añade nuevas capas de identidad, pues permite ampliar este repertorio personal de recursos. Pero este proceso no está exento de tensiones y desafíos, relacionados justamente con la coherencia $-\mathrm{o}$ no- con las creencias y valores sobre la escritura previamente forjados (Lillis, 2013). Por esto, las elecciones discursivas de los escritores en la construcción de sus textos constituyen en sí mismas declaraciones de identidad, materializadas en convenciones que el autor reproduce $-o$ se abstiene de reproducir- según las creencias y valores que ha forjado sobre la escritura.

\section{Identidad e intertextualidad}

Dado que la incorporación de fuentes en la escritura académica es parte esencial de la construcción social del conocimiento y de las prácticas letradas de cualquier comunidad disciplinar, el estudio de la intertextualidad constituye una forma de contribuir a la teoría de la identidad del escritor (Ivanič, 1998). El término intertextualidad es introducido por Kristeva (1986) para nombrar el diálogo establecido entre los diferentes textos, en el entendido de que los preexistentes permean y se conectan con los nuevos. Posteriormente, Fairclough (1992) utiliza el término para aludir a todo tipo de relaciones, 
demarcadas explícitamente o no, que determinado texto establece con otros, y opta por denominar "intertextualidad manifiesta" a los casos en que otros textos son rastreables y abiertamente utilizados para la construcción de uno nuevo.

En la academia, la intertextualidad manifiesta se representa mediante marcas de citación para reconocer expresamente que determinadas ideas son de autoría ajena, y su organización y presentación depende del formatos de citación utilizados por diferentes disciplinas, ya sea en normas (p.e. APA, Chicago, MLA) o en convenciones particulares de revistas o editoriales que adscriben a las lógicas de estas diversas normas. Estas marcas cobran relevancia para la teoría de la identidad puesto que el acto de escritura constituye una declaración de identidad doble (Ivanič, 1998); como un pensador de lo que se dice y como usuario de los recursos lingüísticos elegidos para comunicarlo.

\section{Opciones discursivas de la intertextualidad: estilo e integración}

Cada idea referenciada en un texto puede presentarse mediante un discurso de estilo directo - usando comillas para ilustrar su literalidado indirecto o paráfrasis -adaptando el enunciado original del autor citado y utilizando palabras propias para acomodarlo a la situación comunicativa- (Reyes, 1993). Este mecanismo de inserción de citas relacionado con el estilo ha sido abordado por varios autores, entre ellos Massi (2005). Por su parte, Swales (1990) establece una diferenciación entre citas integrales -el autor de referencia aparece expreso en la oración-y no integrales -el autor citado se presenta entre los paréntesis de referencia- (ver operacionalización de estas categorías en el Marco metodológico).

La interpretación sobre la correspondencia entre niveles de "flexibilidad intertextual" y la selección de estilo de citación ofrecida por Badenhorst (2019) ilustra que, aunque el estilo y la integración de las citas constituyen recursos formales de inclusión de ideas ajenas, lejos de ser neutros, aportan información sobre la construcción identitaria del escritor, cómo se posiciona ante su comunidad y qué imagen proyecta. Reyes (1993) explica que el estilo indirecto involucra 
la interpretación del citador, mientras que en los escritos académicos las citas directas encarnan cierta autoridad y por tanto se conserva su reproducción literal. Análogamente, Swales (1990) puntualiza que la decisión de uso de citas integrales o no integrales concierne a la intención de otorgar mayor importancia al autor citado (integrales) o al contenido de la idea (no integrales).

La relación entre aspectos formales de la citación y cuáles de estos son más o menos privilegiados según disciplinas ha sido bastante explorada. Hyland (1999) ha reportado los avances más significativos analizando 80 artículos contrastivamente, utilizando técnicas de lingüística de corpus y entrevistas a informantes expertos. Sus análisis muestran que en las disciplinas de Biología, Ingeniería Electrónica, Ingeniería Mecánica y Física predominan fuertemente las citas no integrales, mientras que en Marketing, Lingüística Aplicada y Sociología, aunque se mantiene esa predominancia, hay una distribución más equilibrada con las integrales. En Filosofía, no obstante, la predominancia es de las citas integrales, hallazgo concordante con el de Castro y Sánchez (2016) en tesis doctorales de esta área. Respecto al estilo, se advierten mayores diferencias: en las cuatro disciplinas de áreas científicas no aparece ninguna cita directa, mientras que en las Ciencias Sociales sí, aunque son poco frecuentes. Estas tendencias reflejarían "las actividades sociales, estilos cognitivos y creencias epistemológicas de cada comunidad disciplinar" (Hyland, 1999, p. 30, traducción propia).

Estudios posteriores en español tienden a corroborar estos hallazgos. Bolívar (2005) encuentra que en artículos de Artes y Letras muy escasamente utilizan la función "citas de apoyo" (estilo indirecto) y que, al contrario, Letras prefiere las "citas integrales" (estilo directo). En artículos de Comunicación Social, Lingüística, Psicología y Geografía, predominan las "citas de parafraseo" (estilo indirecto). García Negroni (2008) muestra que el estilo directo aparece rara vez en artículos de Medicina y Geología, mientras que es más frecuente en Lingüística e Historia. Sabaj y Paéz (2011) identifican que el estilo directo predomina en artículos de Filosofía y que está totalmente ausente en artículos de Terapia Psicológica, que solo citan indirectamente. En artículos de Lingüística, en tanto, la cita directa está presente pero en mucha menor medida que la indirecta. Sobre la integralidad, los artículos 
de Filosofía distribuyen sus citaciones casi igual entre integrales y no integrales; en Terapia Psicológica, en cambio, las no integrales predominan abrumadoramente; y en Lingüística, la distribución es más equilibrada, pero con predominancia de las no integrales. Fahler y colegas (2019) estudiaron textos de pregrado de Historia y Letras, y encontraron una mayor incidencia del discurso directo en esta última disciplina. En suma, las decisiones de uso referentes al estilo e integración en la inserción de otras voces al texto propio constituyen declaraciones de identidad, socialmente construida, de los escritores que también se relacionan con identidades disciplinares que develan formas de construir conocimiento.

\section{Marco metodológico}

\section{Contexto y diseño del estudio}

Este estudio se realizó en una universidad chilena altamente selectiva con énfasis en investigación cuyo programa doctoral en Educación tiene más de tres décadas. Durante el primer año y medio del programa, los estudiantes elaboran durante tres cursos semestrales la revisión sistemática de la literatura (RSL) (Feak \& Swales, 2009) en torno al posible tema de tesis, lo que les permite acercarse de manera guiada al campo de estudio y servirá como base para edificar su futura investigación doctoral. El primer curso realiza un acercamiento a la búsqueda en bases de datos y exposición descriptiva de resultados del corpus. El entregable final es una revisión breve y expositiva de aproximadamente 10 artículos analizados. El segundo curso profundiza en la escritura de la introducción del artículo RSL y acompaña una primera redacción de patrones de resultados levantados del corpus, punto al que llega el entregable final. El tercer curso retoma lo escrito en los anteriores y avanza hacia la discusión. Su entregable final es una versión completa y publicable de la RSL. Los tres cursos contemplan como mínimo dos instancias de retroalimentación del docente y ejercicios de revisión entre pares. Esta estructura de cursos facilita un estudio continuado de la construcción de un texto, que implica la iteración de la escritura sobre una misma base que va modificándose 
conforme el participante edita y ajusta su escrito, al tiempo que avanza en su formación.

Se plantea un diseño longitudinal cualitativo, de alcance exploratorio. Se organizan cuatro casos de estudio sobre la evolución y las motivaciones declaradas por los escritores de las formas de citación en la reiteración de un mismo texto, con miras a vincular lo que el escritor hace-la forma en que cita-y lo que es -quien escribe(Ivanič, 1998). Para esto, se estudió una muestra intencionada de cuatro doctorandos chilenos, de trayectorias académicas variadas, durante sus primeros dos años de formación doctoral. Para mantener un contexto académico compartido, los participantes se seleccionaron procurando que fueran ingresantes del mismo año, mismo programa y misma institución. Se cuidó de tener una muestra equitativa de hombres y mujeres, y que la lengua materna fuera el español, lengua en que escriben la RSL. Las características de los participantes se especifican en la Tabla 1.

Tabla 1 - Características de los casos

\begin{tabular}{llll}
\hline $\begin{array}{l}\text { PARTICIPANTE } \\
\text { (SEUDÓNIMO) }\end{array}$ & SEXO & EDAD & DISCIPLINA DE ORIGEN \\
\hline SARA & Femenino & 34 & Biología \\
ANDY & Masculino & 28 & Física \\
PEPITA & Femenino & 33 & Economía \\
PIERRE & Masculino & 28 & Matemáticas y física \\
\hline
\end{tabular}

Recolección de datos

Al culminar cada semestre, se solicitó a los participantes la versión del texto entregada en el curso correspondiente. Los archivos fueron anonimizados e identificados con seudónimos. Se contó con un total de tres versiones por participante, es decir 12 documentos: un corpus de 14.708 palabras.

Una vez finalizadas las tres entregas, se entrevistó a cada escritor. El instrumento semiestructurado fue sometido a juicio de un experto en 
el área y modificado según sus observaciones. En estas entrevistas, se le mostró a cada participante sus frecuencias de uso de los distintos tipos de cita para recoger sus interpretaciones sobre el proceso y enriquecer las fuentes de análisis. La Tabla 2 ilustra preguntas personalizadas, según los resultados del análisis:

Tabla 2 - Ejemplos de preguntas

\begin{tabular}{ll}
\hline Participante & Ejemplo \\
\hline SARA & $\begin{array}{l}\text { La mayoría de las veces citaste indirectamente, ¿existe alguna } \\
\text { razón por la que consideres preferible hacerlo así? }\end{array}$ \\
ANDY & $\begin{array}{l}\text { Podría pensarse que prefieres las citas no integradas, ¿es así?, } \\
\text { ¿existe alguna razón por la que cites siempre de esta manera? }\end{array}$ \\
PEPITA & $\begin{array}{l}\text { Solo citaste de manera directa, y en las } 3 \text { versiones, a la UNESCO. } \\
\text { ¿Hay alguna razón por la cual hayas decidido que esta referencia } \\
\text { merecía ser presentada con un estilo distinto a las demás? }\end{array}$ \\
& $\begin{array}{l}\text { Conforme avanzan las versiones incrementas el uso de la cita } \\
\text { integral, ¿a qué le atribuyes eso? }\end{array}$ \\
\hline
\end{tabular}

Las entrevistas fueron grabadas en audio y transcritas usando un procesador de textos en ortografía estándar, anonimizadas e identificadas con seudónimos. El proceso de recolección de los datos (Figura 1) contó con supervisión y aprobación del Comité de Ética de la institución.

Figura 1 - Modelo de recolección de datos

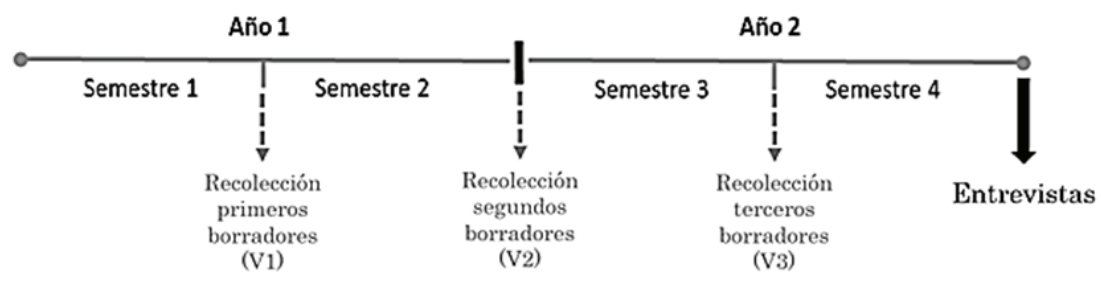




\section{Análisis de datos}

Para responder a las dos primeras preguntas - ¿cómo los estudiantes doctorales distribuyen sus formas de citación en una misma práctica letrada? y ¿qué cambios tienen estas distribuciones a lo largo de diferentes versiones del texto producidas durante un año y medio de formación doctoral? - se utilizaron herramientas de análisis del discurso para clasificar el estilo e integración de las citas del corpus. Se analiza la introducción porque constituye el momento en que el escritor establece el territorio y define un nicho dentro del campo de estudio (Swales, 1990), y para ello debe dialogar con otros autores, lo que implica decisiones de alineamiento teórico y epistemológico. Lo anterior entendiendo que el género respondía a la estructura tradicional IMRD (introducción, método, resultados y discusión); y, por ende, la introducción incluía aspectos tales como antecedentes y marco teórico.

Primero, se identificaron y cuantificaron las citas, que sumaron un total de 275 , distribuidas como presenta la Tabla 3.

Tabla 3 - Distribución de citas

\begin{tabular}{llccc}
\hline & \multicolumn{3}{c}{ Total citas } \\
\cline { 1 - 2 } Versiones texto & V1 & & V2 & V3 \\
\cline { 1 - 2 } Pierre & 19 & & 37 & 39 \\
Sara & 23 & & 23 & 16 \\
Andy & 18 & & 20 & 23 \\
Pepita & 20 & & 19 & 19 \\
\hline
\end{tabular}

Segundo, cada cita se clasificó según estilo e integración, categorías operacionalizadas y ejemplificadas en la Tabla 4 partiendo de las definiciones de Swales (1990) y Reyes (1993). 
Tabla 4 - Categorías de clasificación y ejemplos

\begin{tabular}{|c|c|c|}
\hline & Integral & No integral \\
\hline & $\begin{array}{l}\text { Alusión explícita } \\
\text { al autor; este es un } \\
\text { participante en la } \\
\text { cláusula donde se refiere } \\
\text { a sus ideas. }\end{array}$ & $\begin{array}{l}\text { Mención del autor dentro de la } \\
\text { referencia. }\end{array}$ \\
\hline $\begin{array}{l}\text { Cita directa } \\
\text { Reproducción } \\
\text { literal de las } \\
\text { palabras de otro } \\
\text { autor. }\end{array}$ & $\begin{array}{l}\text { Ejemplo cita directa - } \\
\text { integral } \\
\text { Tuan (2014) afirma } \\
\text { que la "experiencia } \\
\text { está compuesta } \\
\text { de sentimientos y } \\
\text { pensamientos" (p.10) } \\
\text { [cV1] }\end{array}$ & $\begin{array}{l}\text { Ejemplo cita directa - no } \\
\text { integral } \\
\text { El ODS4 propone "garantizar una } \\
\text { educación inclusiva, equitativa } \\
\text { y de calidad y promover } \\
\text { oportunidades de aprendizaje } \\
\text { durante toda la vida para todos" } \\
\text { (UNESCO, 2017, p.12) [lV3] }\end{array}$ \\
\hline $\begin{array}{l}\text { Cita indirecta } \\
\text { Parafraseo de las } \\
\text { palabras de otro } \\
\text { autor referenciado. }\end{array}$ & $\begin{array}{l}\text { Ejemplo cita indirecta - } \\
\text { no integral } \\
\text { Borko (2004) estudió qué } \\
\text { hallazgos y preguntas } \\
\text { se habían investigado } \\
\text { en la literatura sobre el } \\
\text { desarrollo profesional } \\
\text { docente en matemática. } \\
\text { [pV2] }\end{array}$ & $\begin{array}{l}\text { Ejemplo cita indirecta - no } \\
\text { integral } \\
\text {...recientes investigaciones } \\
\text { sugieren que ayudar a los } \\
\text { estudiantes a lograr los resultados } \\
\text { de aprendizaje deseados mediante } \\
\text { actividades de laboratorios es un } \\
\text { proceso altamente complejo (Avi } \\
\text { Hofstein \& Lunetta, 2004) [fV1] }\end{array}$ \\
\hline
\end{tabular}

La codificación completa fue revisada mediante auditoría experta. Para obtener números normalizados y ver cómo se distribuían las categorías entre las versiones de un mismo participante, se dividió la recurrencia de cada categoría en el número total de citas incluidas en la respectiva versión.

Concluido este análisis, y para atender a la última pregunta-¿qué razones atribuyen los estudiantes a estas elecciones y preferencias de uso?-, se analizaron las entrevistas desde una perspectiva hermenéutica (Kvale, 1996) para interpretar las elecciones de los individuos en relación con sus textos. 


\section{Resultados}

A continuación se presentan los resultados por caso, mediante una gráfica que atiende a las primeras dos preguntas de investigación. Posteriormente, se indagan las motivaciones emergidas en las entrevistas para responder a la tercera pregunta.

\section{Pierre}

El Gráfico 1 ilustra los estilos e integralidad de las citas de Pierre a través del tiempo.

\section{Gráfico 1 - Pierre: distribución de citas}

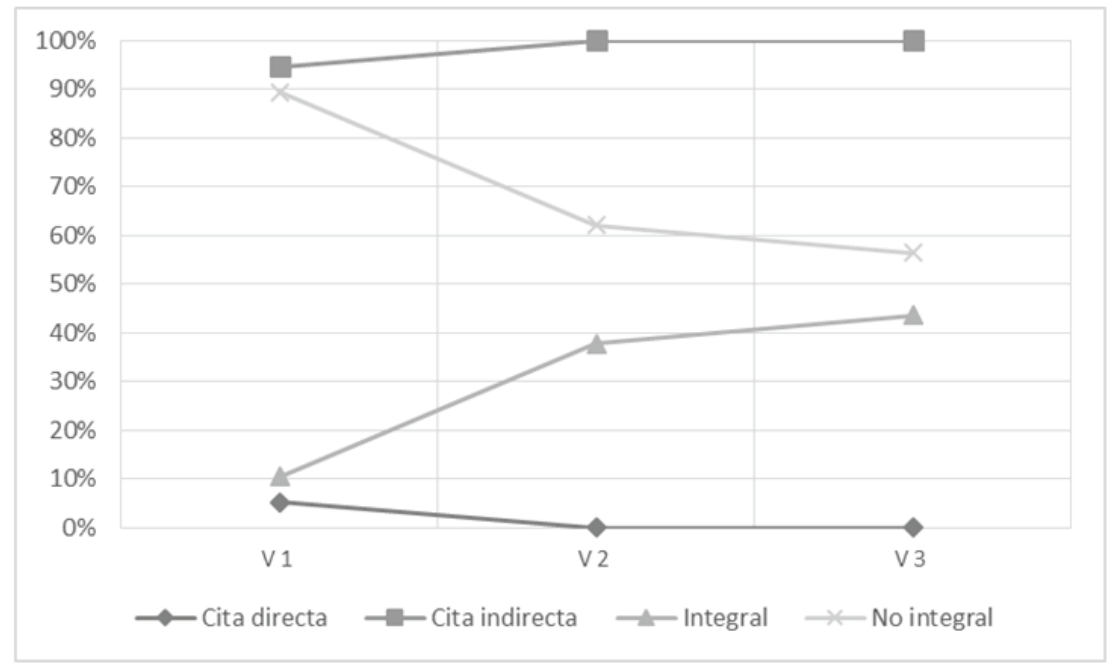

Prácticamente todas las citas en las tres versiones son de estilo indirecto; únicamente en la primera versión se reconoció una cita directa, que luego se omite. En contraste, aunque hay un predominio de citas no integrales entre las versiones, con el tiempo se advierte un aumento del uso de integrales.

En su entrevista, al observar la distribución de sus citas según el estilo, Pierre relaciona su inclinación a la paráfrasis con una costumbre 
adquirida al momento de escribir y que considera "más sencillo". Además, explica que, por lo general, está citando fuentes que lee en inglés, de manera que parafrasear las ideas que ha leído y adecuarlas a su texto le resulta más fácil que correr el riesgo de hacer una traducción literal que no sea fiel al original: "tampoco sé si mi traducción es correcta, entonces ponerle 'traducción propia' siento que no es tan bueno... mejor dar a entender lo que yo entiendo por la cita”.

El uso de la primera personal "lo que yo entiendo" cobra mayor resonancia cuando, más adelante, reflexiona sobre la imagen que a su juicio proyecta del estilo directo, a la vez que establece un puente entre la lectura y escritura, y da cuenta de decisiones y de valoraciones sobre los modos de leer los textos y de integrar esas ideas en la construcción de una propuesta propia:

"No me gustan mucho [las citas directas] porque siento que es como un copy-paste. La gracia de la cita también está en cómo la persona que está escribiendo entendió lo otro y lo integra a su texto. Un texto que tiene mucha cita textual también significa que hay un trabajo de no entender y no interpretar lo que se leyó y entendió".

Con esta declaración Pierre expresa la creencia de que el estilo indirecto corresponde a una falta de comprensión de las fuentes o a un menor nivel analítico. Esta creencia es muy interesante, porque probablemente responde a que en su formación inicial y profesional, las citas no suelen ser fuentes de interpretación o hermenéutica, y al contrario, presentan un valor de verdad.

Una reacción distinta es generada con los resultados sobre la integralidad. Sobre la variación entre sus versiones, Pierre confiesa que este cambio responde a que antes desconocía el uso de las citas integrales:

"No es conscientemente, solo lo empecé a leer más. Uno va viendo que se usa el recurso, pero nunca lo había ocupado. Para mí la cita siempre iba entre paréntesis. Pero después resultó bastante útil porque así uno puede poner en contraste dos autores frente a una misma idea o un mismo tema, y eso me ha ayudado también a posicionarme, que era algo que nos pedían [en los cursos del programa]". 
Este descubrimiento producto de la familiarización con las prácticas privilegiadas en su nueva comunidad discursiva, el doctorado en Educación, le permite negociar su identidad como escritor académico y ampliar su repertorio de recursos. Dicha ampliación le resulta "bastante útil" pues le permite resolver la práctica de contrastar posturas, solicitada explícitamente en el programa, y que no parecía ser valorada en su disciplina de origen:

“Acá en Ciencias Sociales resulta más normal citar a un autor particular, mencionarlo, así como 'la idea de alguien'. Porque dos personas pueden opinar diferente sobre un mismo tema. En Ciencias no suele pasar eso. Si tú logras demostrar algo, es poco probable que alguien te lo contradiga".

Pierre establece conexiones entre los usos de integración y las formas de construir el conocimiento que se privilegian en las comunidades disciplinares en las que ha participado, y que ya han sido develadas por la literatura. De este modo, además de ampliar su repertorio de recursos, el participante desarrolla creencias para conectar estos recursos con asuntos epistemológicos.

\section{Sara}

Las distribuciones entre las versiones de Sara muestran un poco más de variabilidad que las de Pierre. Con el tiempo, hay una leve disminución del uso de estilo indirecto, aunque este es predominante $\mathrm{y}$, consecuentemente, un incremento de citas directas. Asimismo, Sara, a diferencia de los demás participantes, es asidua del recurso de integración; incluso en su segunda versión las citas integrales superan el $50 \%$. 
Gráfico 2 - Sara: distribución de citas

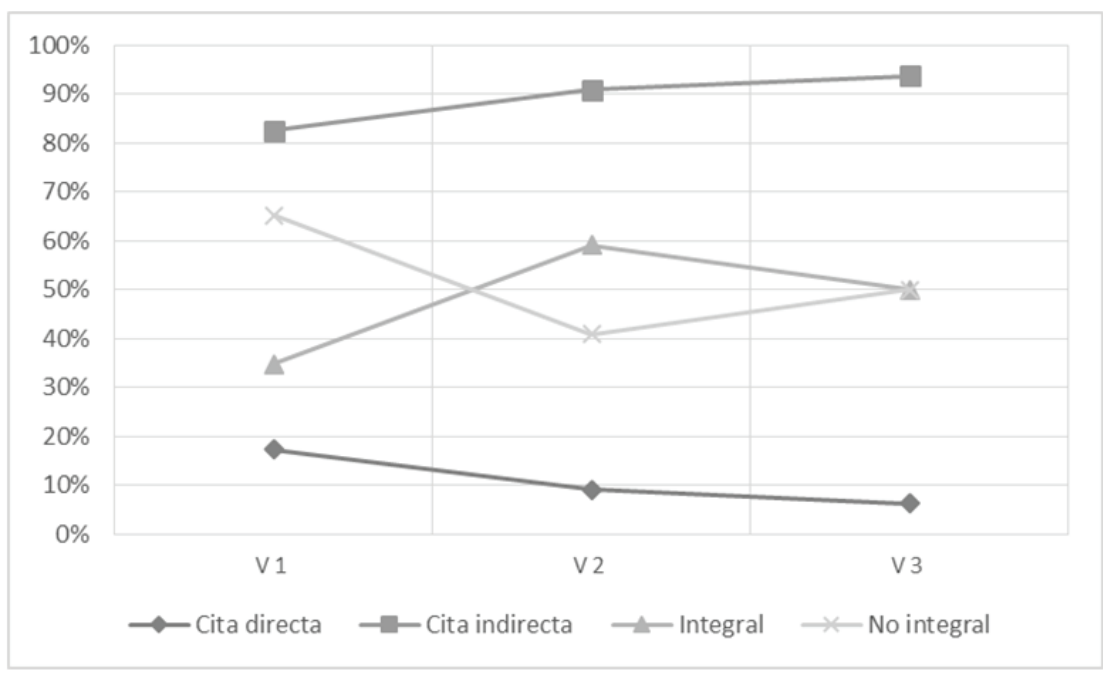

Según lo manifestado en la entrevista, la inclinación por la paráfrasis responde a las prácticas que mantiene de lectura e incorporación de referencias:

"yo creo que tiene que ver con que trabajo mucho los textos. De hecho, yo soy de leer y escribir al mismo tiempo, entonces voy trabajando el parafraseo, de alguna manera, siempre que voy leyendo... Porque siempre me pasa que me gusta una idea pero ¿cómo aporta a lo mío?”

En ella, entonces, la inclinación por la paráfrasis no es consciente, sino producto de sus estrategias de escritura, que combina con una lectura focalizada en la extracción de información que aporte a sus intereses. Distinto a Pierre, Sara, a partir de los textos con los que ha venido familiarizándose, ha forjado la creencia de que el uso de la cita directa se vincula con autores e ideas que poseen una relevancia destacable dentro del tema tratado. Es decir, que suele ser un estilo recurrente para citar autores "clásicos".

Cuando Sara observa la distribución de integralidad, se sorprende de los resultados, pues "hubiera creído que era más de no integral. . . porque en Biología se cita mucho no integral". Consecuentemente, para Sara la "identidad" del investigador en Ciencias Naturales se condice con determinado recurso discursivo, el privilegio de las citas 
no integrales y, por lo tanto, se extraña de no haber actuado acorde a su membresía en su disciplina de origen.

Al respecto, es central observar su desarrollo en la nueva comunidad disciplinar, del que emerge esta negociación. Sara cuenta que en el doctorado ha estado trabajando "cada vez más con filósofos", de manera que es posible que la familiarización con prácticas letradas distintas a las conocidas haya comenzado a permear sus elecciones discursivas $\mathrm{y}$, por ende, su identidad como escritora académica. Indica que no es una elección deliberada y que solo lo hace "cuando le nace", lo que devela cierta identificación no consciente con esta nueva comunidad discursiva mediante la adopción gradual de sus prácticas.

Igual que Pierre, Sara amplía su repertorio de recursos discursivos, lo que se manifiesta en el asombro frente a la tendencia de elección no consciente de las citas no integrales, producto de la socialización con nuevas prácticas. Sara tenía la creencia de mantener un arraigo con sus prácticas discursivas anteriores, y se sorprende al observar la integración de recursos que son más valorados en la nueva comunidad disciplinar.

Andy

Andy presenta una marcada tendencia en el uso de citas indirectas y no integrales. De hecho, prescinde por completo del uso de citas directas; mientras que, como Pierre, va aumentando levemente el uso de citas integrales. 
Gráfico 3 - Andy: distribución de citas

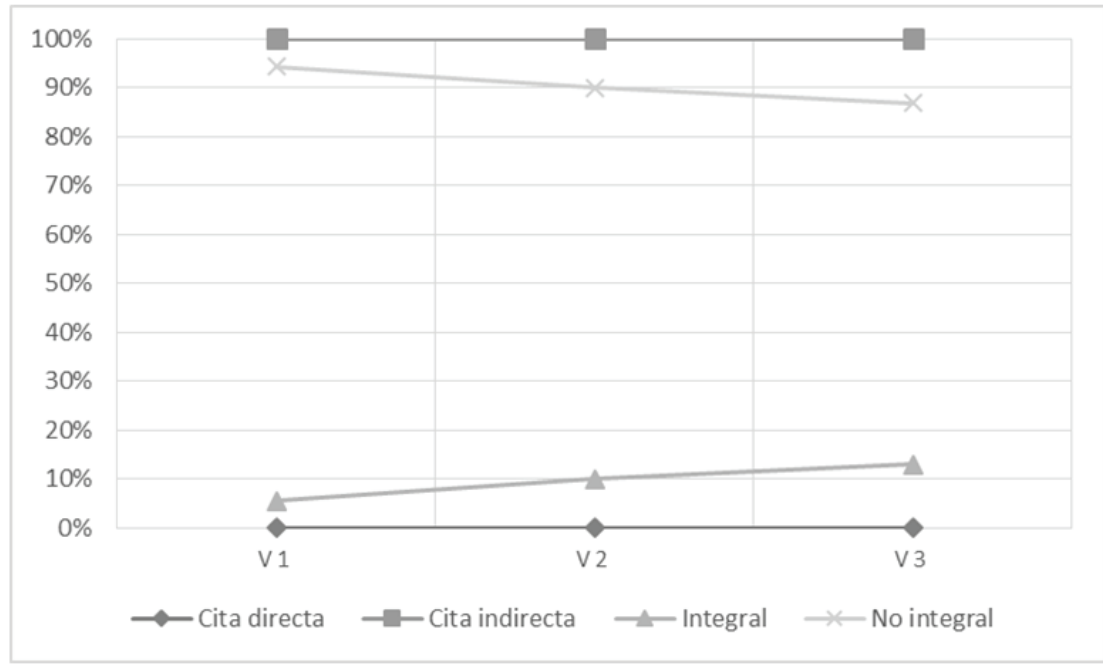

Al ver sus resultados, Andy explica que considera más sencillo adecuar las ideas al propio discurso que hacer uso de fragmentos literales:

"[el estilo directo] implica que uno tiene que organizar mejor ese párrafo para que decante en esa cita textual o cita directa, y eso requiere para mí un proceso cognitivo mayor que el que implica integrar el texto anterior con la cita indirecta y con lo que viene después".

En este sentido, se advierte que su elección de uso responde a la facilidad que la paráfrasis le representa para adecuar las ideas ajenas al discurso propio. Andy, entonces, coincide parcialmente con Pierre, en que considera más sencillo escribir haciendo uso de citas indirectas. No obstante, difieren en la consideración sobre el esfuerzo cognitivo que estos recursos demandan: para Pierre, el uso de la cita directa puede evocar pobreza de comprensión o interpretación y se refiere a formas de leer. Para Andy, su uso demanda "un proceso cognitivo mayor" que la paráfrasis, evaluación que pareciera construir desde una dimensión lingüística: construir una sola oración con sentido que integre las ideas propias y de otros.

Más aún, Andy coincide con Sara al enlazar el estilo directo con la incorporación de posiciones teóricas con las que se alinea o identifica. 
Él explica que "para usar eso, lo único que se me ocurre es que tendría que estar hablando de un tema a nivel más teórico, que requiere una posición teórica específica", como ha visto que se utiliza: "Las citas directas las he leído cuando están haciendo contribuciones más teóricas que empíricas".

De forma similar, sobre su distribución de citas por integración, sin mostrar sorpresa frente al resultado, Andy declara que "no estila" usar formas integrales porque su uso constituye una declaración de compromiso teórico y de alineación con el autor. Además, vincula esta decisión con su "hábito" de parafrasear:

"me gusta tener propiedad del texto y porque, como parafraseo, para poder decir 'este tipo dice tal cosa', uno tiene que estar muy seguro de lo que efectivamente está diciendo ese tipo. Entonces prefiero relajar un poco eso y hacerlo no integral para evitar esa clase de ambigüedad, y porque nunca me siento -yo creo que es mi personalidad- tan seguro de parafrasear a un autor argumentando "este autor dice"”

y luego añade "Es un poco cobarde mi tipo de escritura". Las elecciones de Andy sobre la integración de las citas está condicionada por la creencia de que mediante el estilo integral se establece una declaración de compromiso teórico mayor con el autor que se referencia. Se advierte además una reflexión epistémica del uso de la cita directa, vinculada con la seguridad que él pueda tener de la interpretación de lo que postula un autor para integrarlo explícitamente y visibilizarlo en la oración. Así, Andy, considera lógico haber aumentado el número de citas integrales entre las versiones: "Me hace sentido porque ya tenía más conocimiento, sabía qué decía más un autor que otro". Su sentido de autoridad frente a la comunidad aumenta. Mientras las interpretaciones de Andy muestran altos grados de agencia y control de las decisiones al escribir, sus patrones resultan hondamente alineados con las formas de citación que la investigación ha presentado como privilegiadas en Física, su disciplina de origen, a saber, el predominio de citas indirectas y no integrales. 


\section{Pepita}

Pepita es la única participante que no presenta cambios en estilo e integración durante el periodo estudiado. Ella mantiene una misma cita directa en las tres versiones y en ninguna utiliza citas integrales.

Gráfico 4 - Pepita: distribución de citas

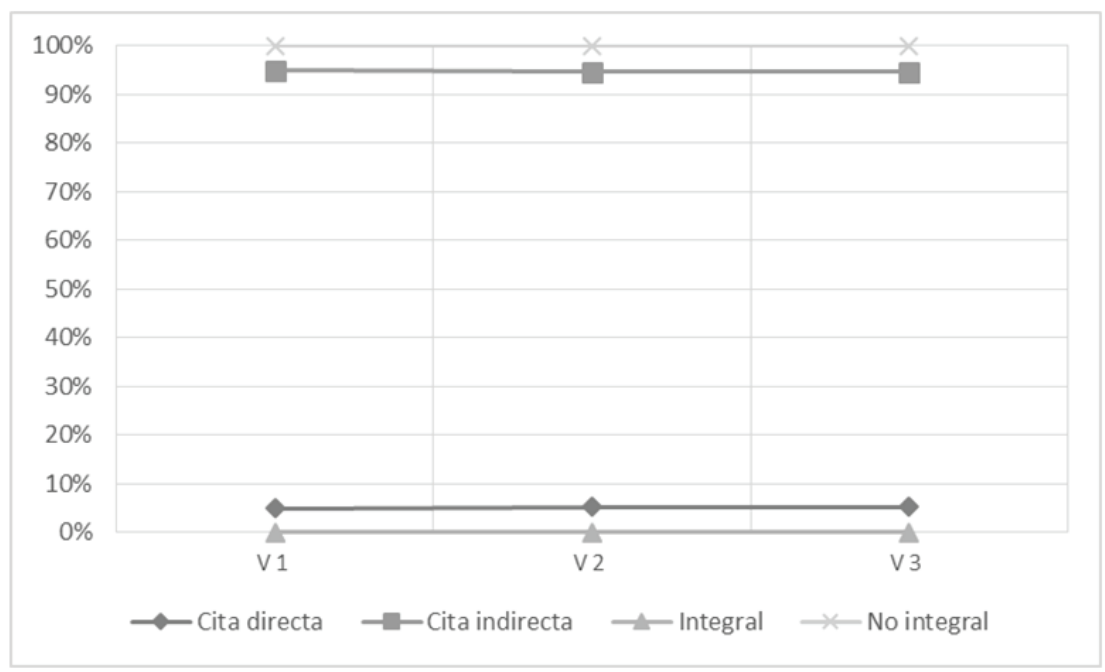

Las respuestas de Pepita sugieren que no comparte la perspectiva sobre la facilidad de parafrasear planteada por Andy. Pese a reconocer que el uso de la paráfrasis es una costumbre sobre la que no había reflexionado, indica que le resulta una tarea compleja:

"cuando a ti te cuentan una historia, tú tratas de contarla lo más parecido a lo que te la contaron. Entonces cómo después uno se abstrae de eso y dice 'ya, esta persona me dijo esto y tengo que decir esto pero no lo puedo decir como lo dijo él”".

Pese a esta dificultad, prefiere el uso indirecto porque, como Pierre y Andy, es crítica con el uso asiduo del estilo directo: "Pensando como si yo estuviera leyendo un texto que no es mío, y veo muchas citas directas, yo diría 'uy, este gallo llegó y copió"'. Se advierte nuevamente la vinculación entre el recurso y la proyección de determinada identidad académica, además de hacer eco a la vinculación ya establecida por 
Pierre entre la cita directa y la carencia de un trabajo interpretativo o de menor demanda cognitiva.

En contraste con esta creencia sobre la identidad proyectada mediante el uso de citas directas, Pepita recuerda que al iniciar el doctorado usaba más la forma integral, pero su tutor le recomendó evitarlo, porque su abuso hacía "muy tediosa la lectura". Así, Pepita evita proyectarse como una narradora "tediosa" y negocia su identidad mediante elecciones discursivas que utiliza para adecuarse a lo más valorado por la comunidad académica. Es, además, un acto de cesión:

"Me gusta más [la cita] integral, siento que me va a quedar mucho más en la cabeza. . . Pero, para escribir, ya lo hago automático, escribo no integral, porque me dijeron que lo hiciera así, que no hiciera integral. Trato de disminuirlo al mínimo".

Aunque como lectora sostiene cierta creencia sobre este recurso, decide reproducir determinada elección para proyectar una identidad como escritora académica que parece más valorada en ese contexto. En esta concepción se aleja de Sara y Andy, quienes vinculan el recurso a prácticas disciplinares.

Asimismo, cuando Pepita nota que la mayoría de las citas integrales corresponden a tres autores específicos, añade: "Puede ser que es cuando estoy más cercana a ellos, cuando los he leído más. Parece que sí, ¿sabes? Puede ser, porque son los que más he trabajado, más he leído, que más comparto". Es decir que, semejante a Andy, Pepita enlaza el uso directo con la alineación y seguridad de conocimiento sobre determinados autores.

\section{Discusión}

Las distribuciones de las formas de citación de los participantes, en términos de estilo e integración, muestran marcadas tendencias de uso y pocos cambios en el tiempo. Sobre los cambios temporales, cabe considerar que este trabajo tiene una amplitud de tan solo tres semestres, período en el que se pudo rastrear la historia textual de un solo producto escrito, pero que no necesariamente permite dar cuenta 
de los cambios experimentados al participar progresivamente en una comunidad disciplinar nueva. En ese sentido, recién al tercer año los estudiantes se sumergen formalmente en su proceso de investigación independiente como tesistas; por tanto, es probable que una perspectiva mayor del tiempo permita observar aún mayores diferencias adquiridas con la experiencia. Por esto, cabe destacar que los resultados corresponden a una etapa temprana del proceso de socialización con una nueva comunidad discursiva y sus asociadas prácticas de privilegio. A medida que se vayan adentrando en la escritura de su tesis y en otras prácticas letradas propias de la academica, como los artículos de investigación, estas tensiones y negociaciones identitarias pueden cobrar otros matrices o acentuarse para continuar modificando las elecciones discursivas de los participantes.

Con ánimo comparativo entre escritores, el uso de citas indirectas es predominante. Desde una visión intra-participante, el uso del estilo no sufre ninguno o bien mínimos cambios entre las versiones de cada escritor. Respecto a la integración es perceptible la preferencia por el estilo no integral, aunque esta tendencia es menos notoria en comparación al estilo de citación.

En términos de adscripción disciplinar, hay perfiles interesantes. Los resultados de estilo muestran, en todos los participantes (provenientes de una formación profesional previa en Matemáticas, Biología, Física y Economía), una adscripción implícita al estilo indirecto, preponderante en las áreas científicas (Hyland, 1999), que probablemente responda a las formas en la que fueron enculturados y con las que aprendieron a construir conocimiento. Al ser familiares, estas formas discursivas tienden a ser calificadas por los participantes como "hábitos", “costumbres" o usos más sencillos, pues se encuentran incorporadas en sus repertorios de recursos discursivos. También destaca que algunos vinculen las citas con "copia" o "falta de comprensión", aunque la investigación muestra que esta práctica es frecuente en las Humanidades, en áreas como Arte, Literatura, Letras o Filosofía (Bolívar, 2005; García Negroni, 2008; Fahler et al., 2019), en las que probablemente funciona como fuente para análisis hermenéuticos.

Por otro lado, las dudas y juicios en torno al uso de la cita directa son también un factor que se vincula al difícil desarrollo de una voz 
autoral, una problemática que plantea Zavala (2011): sus participantes conciben la intertextualidad académica como falta de originalidad. En tanto, Ivanič (1998) problematiza la idea simplista de plagio versus originalidad. La copia no corresponde solamente a citar sin atribuir, y hay un límite difuso entre "la imitación" del discurso académico de la comunidad a la que los doctorantes aspiran a pertenecer, el plagio y la emergencia de una voz de autoridad que recurre a la intertextualidad. Para la autora, apegarse extremadamente a las fuentes podría ser incluso una estrategia imitativa que conduzca a adquirir una voz propia legítima en esta nueva comunidad; se trata más bien de una gradación difusa desde "la copia, la imitación, hasta adquirir la adquisición de un nuevo discurso" (Ivanič, 1998:195). Se atribuye erróneamente de esta forma un valor superior a "la originalidad", idea de sentido común que encuentra eco en algunos de los participantes.

Sobre la integralidad, en tres de los cuatro participantes hay una alta predominancia de citas no integrales, convergentemente con lo sugerido por Hyland (1999) para las disciplinas científicas. Resulta revelador que uno identifica la función de las citas integrales en su nueva comunidad discursiva para establecer un nuevo tipo de relación con la fuente; a saber, enfrentar o contrastar perspectivas entre autores. En tanto, otra se sorprende de haber utilizado citas integrales, lo que en parte relaciona con su trabajo con la Filosofía de la Educación, área con la cual parece establecer una identificación hasta entonces no consciente.

En síntesis, estos resultados se relacionan con los procesos de construcción y negociación de identidad académica de los participantes, que pueden agruparse en dos tendencias no excluyentes: (i) "hábitos" relacionados con sus identidades previas, y (ii) estrategias desplegadas a partir de creencias sobre la escritura, que se modifican conforme se participa de nuevas comunidades.

Primero, se observa una reproducción, a veces inconsciente, de prácticas incorporadas durante la formación de sus identidades académicas previas, que los participantes identifican como "hábitos", y que la literatura califica como tendencias disciplinares. La reproducción de estas prácticas e identificaciones no conscientes con comunidades discursivas de las que se ha participado podría parcialmente explicar 
la ausencia de cambios evidentes en el uso de este recurso durante el periodo estudiado.

Segundo, las respuestas de los participantes sobre las distribuciones encontradas revelan creencias que han desarrollado sobre la escritura que se traducen en elecciones estratégicas de uso. Es decir, realizan elecciones para mantener una fluidez en el discurso y proyectar la identidad de un escritor crítico, que comprende y trabaja sus textos y analiza las ideas ajenas. Son decisiones influenciadas, por una parte, por lo que ellos consideran se privilegia en un texto académico acorde con la imagen que tienen de un experto y que desean presentar frente al lector, una suerte de identidad deseada (Zavala, 2011); y, por otra, por las creencias que han forjado desde su posición de lectores académicos. Esta estrategia coincide con otros estudios (Calle-Arango et al., 2021, Harwood \& Petrić, 2012). Asimismo, las creencias de los participantes respecto del parentesco entre el recurso y una u otra comunidad discursiva, y como consecuencia con la imagen que su uso proyecta, recuerda lo hallado entre los escritores de Jomaa y Bidin (2019), quienes también identificaron que la integralidad está condicionada por el tipo de fuente referenciada y la intención que se pretende generar con la información que se transmitirá.

Además, especulaciones como las relativas a la proyección de identidades determinadas, aunadas a las reflexiones sobre la facilidad del uso de la paráfrasis como forma de adecuar y recontextualizar las ideas, y en sintonía con la preocupación sobre la necesidad de tomar distancia frente a las palabras originales de la fuente cuando se utiliza el estilo indirecto, permiten identificar que los participantes apuntan a una "alta flexibilidad intertextual" (Badenhorst, 2019) en su construcción de identidad académica.

En suma, los participantes sugieren, por un lado, una reproducción de ciertos recursos, pero también valores, privilegiados en su comunidad de formación profesional. Pero estos, por otro lado, se tensionan con aquellos usos y valores privilegiados en la nueva cultura escrita con la que están socializando, que se materializan en decisiones conscientes de adopción o elección estratégica de ciertos usos. 


\section{Conclusiones}

El presente estudio se formuló las preguntas ¿cómo los estudiantes doctorales distribuyen sus formas de citación en una misma práctica letrada?, ¿qué cambios tienen estas distribuciones a lo largo de diferentes versiones del texto producidas durante un año y medio de formación doctoral? y ¿qué razones atribuyen los estudiantes a estas elecciones y preferencias de uso? El análisis de las formas de citación en términos de estilo e integración mostró preferencias semejantes entre los participantes, así como pocos cambios en el tiempo. Los hallazgos permiten identificar vínculos con patrones discursivos previamente descritos, pero las entrevistas permiten también mostrar que tales elecciones responden a procesos de construcción y negociación identitaria más complejos.

Entonces, es posible observar la interacción entre creencias, negociación de elecciones y ampliación de recursos discursivos personales que dan cuenta del desarrollo de una nueva capa de identidad académica en la disciplina de Educación, distinta de las disciplinas y profesiones previas de los participantes. Este complejo entramado ratifica cómo la participación en múltiples contextos conlleva a una identidad heterogénea (Ivanič, 1998; Zavala, 2011) y a una mayor flexibilidad de la identidad académica según el contexto y el lector conforme se amplía el repertorio personal de elecciones discursivas.

En virtud de lo anterior, los métodos y hallazgos presentados en esta investigación contribuyen a complejizar la relación texto-escritor, particularmente en estudios interesados en la identidad discursiva, tensiones involucradas en la construcción de identidades heterogéneas y sus negociaciones en el contexto de la formación doctoral.

\section{Agradecimientos}

Esta investigación se realizó con el apoyo de la Agencia Nacional de Investigación y Desarrollo de Chile, ANID, folio 21180042. 


\section{Conflicto de intereses}

Los autores declaran no tener ningún conflict de interesses.

\section{Contribución de los autores}

Nosotras, Lina Calle-Arango, Natalia Ávila Reyes y Alejandra Meneses, declaramos por la presente que no tenemos ningún conflicto de intereses en este estudio. Todas participamos en la conceptualización del estudio, la metodología, el análisis formal de datos y la redacción del presente escrito.

\section{Referencias bibliográficas}

Araújo, A. (2006). Práticas discursivas em conclusões de teses de doutorado. Linguagem Em (Dis)Curso, 6(3), 447-462.

Badenhorst, C. (2019). Literature reviews, citations and intertextuality in graduate student writing. Journal of Further and Higher Education, 43(2), 263-275. https://doi.org/10.1080/0309877X.2017.1359504

Barton, D., \& Hamilton, M. (2000). Literacy Practices. In D. Barton, M. Hamilton, \& R. Ivanič (Eds.), Situated Literacies. Reading and writing in context (pp.7-15). London: Routledge.

Bazerman, C. (2004). Intertextuality: How texts rely on other texts. In C. Bazerman, \& P. Prior (Eds.), What Writing Does and How It Does It: An Introduction to Analyzing Texts and Textual Practices (pp. 83-96). EUA: Lawrence Erlbaum Associates. https://doi. org/10.4324/9781410609526

Bolívar, A. (2005). Tradiciones discursivas y construcción del conocimiento en las humanidades. Signo y Seña, (14), 67-91.

Burgess, A., \& Ivanič, R. (2010). Writing and Being Written: Issues of Identity Across Timescales. Written Communication, 27(2), 228 -255. https://doi.org/ 10.1177/0741088310363447

Calle-Arango, L., \& Ávila Reyes, N. (2020). Alfabetización académica chilena: revisión de investigaciones de una década. Literatura y Lingüística, 41, 455-482. https://doi.org/10.29344/0717621X.41.2280

Calle-Arango, L., Ávila Reyes, N., \& Meneses, A. (2021). Construcción y transformaciones de las identidades académicas de estudiantes doctorales mediante la citación. Íkala, 26(2), 341-356. https://doi. org/10.17533/udea.ikala.v26n02a12

Canata, F., Çapkın, C., Doğan, G., Sünger, B., \& Oynak, E. (2017). Citation Analysis of Dissertations Completed at Istanbul University 
Information and Records Management Department. Turkish Librarianship, 31(1), 31-46.

Castelló, M. (2016). Escribir artículos de investigación. Aprender a desarrollar la voz y la identidad del investigador novel. In G. Bañales, M. Castelló, N. Vega (Eds.). Enseñar a leer y escribir en la educación superior. Propuestas educativas basadas en la investigación (pp. 211-232). Libros digitales. https://doi.org/004.

Castelló, M., McAlpine, L., Sala-Bubaré, A., Inouye, K., \& Skakni, I. (2020). What perspectives underlie 'researcher identity'? A review of two decades of empirical studies. Higher Education. https://doi. org/10.1007/s10734-020-00557-8

Castro, M.C., \& Sánchez, M. (2016). La formación de investigadores en el área de humanidades: Los retos de la construcción de la voz autoral en la escritura de la tesis de doctorado. Signos, 49(S1), 30-51. https:// doi.org/10.4067/S0718-09342016000400003

Chiware, E., \& Becker, D. (2018). Citation Patterns of Conference Proceedings in Master's and Doctoral Studies: A Case Study of Information Technology and Systems. SAGE Open, 8(2). https://doi. org/10.1177/2158244018770496

Choi, Y., Bouwma-Gearhart, J., \& Ermis, G. (2021). Doctoral Students' Identity Development as Scholars in the Education Sciences: Literature Review and Implications. International Journal of Doctoral Studies, 16, 089-125. https://doi.org/10.28945/4687

Chois, P., \& Jaramillo, L. (2016). La investigación sobre la escritura en posgrado: estado del arte. Lenguaje, 44(2), 227-259.

Dávila, M. (2012). Tendencias internacionales en posgrados. Integración $y$ Conocimiento, 1, 18-26.

Difabio De Anglat, H., \& Álvarez, G. (2019). Estrategias retóricas del capítulo introductorio de tesis doctorales en Educación. Íkala, 1(24), 69-84. https://doi.org/10.17533/udea.ikala.v24n01a03

Fahler, V., Colombo, V., \& Navarro, F. (2019). En búsqueda de una voz disciplinar: intertextualidad en escritura académica de formación en carreras de humanidades. Calidoscopio, 17(3), 554-574. https://doi. org/10.4013/cld.2019.173.08

Fairclough, N. (1992). Discourse and Social Change. Cambridge: Polity Press. https://doi.org/10.2307/2074659

Fazel, I., \& Shi, L. (2015). Citation behaviors of graduate students in grant proposal writing. Journal of English for Academic Purposes, 20, 203-214. https://doi.org/10.1016/j.jeap.2015.10.002

Feak, C., \& Swales, J. (2009). Telling a Research Story: Writing a Literature Review. EUA: University of Michigan Press. 
Fisher, R., Brock, C., Frahm, T., Van Wig, A., \& Gillis, V. (2020). Reflections on writing and identity: exploring the role of qualifying exams in the sociocultural development of doctoral students. Studies in Continuing Education, 42(3), 365-380. https://doi.org/10.1080/0 158037X.2019.1661237

García Negroni, M. (2008). Subjetividad y discurso científico-académico. Acerca de algunas manifestaciones de la subjetividad en el artículo de investigación en español. Signos, 41(66), 9-31. https://doi. org/10.4067/S0718-09342008000100001

Harwood, N., \& Petrić, B. (2012). Performance in the citing behavior of two student writers. Written Communication, 29(1), 55-103.

Hyland, K. (1999). Disciplinary Discourses. Social Interactions in Academic Writing. EUA: University of Michigan Press. https://doi. org/10.1017/CBO9781107415324.004

Inouye, K., \& McAlpine, L. (2019). Developing Academic Identity: A Review of the Literature on Doctoral Writing and Feedback. International Journal of Doctoral Studies, 14, 1-31.

Ivanič, R. (1998). Writing and identity. The discoursal construction of identity in academic writing. New England: Lancaster University.

Jomaa, N., \& Bidin, S. (2019). Variations in the citation use and perceptions in writing the literature review by EFL postgraduates. Eurasian Journal of Applied Linguistics, 5(3), 441-460. https://doi. org/10.32601/ejal.651398

Kristeva, J. (1986). The Kristeva Reader. In: Toril Moi, Ed. PsycCRITIQUES. Columbia University Press. https://doi.org/10.1037/027283

Kvale, S. (1996). The Interviews. An Introduction to Qualitative Research Interviewing. London: Sage Publications.

Lillis, T. (2013). The sociolinguistics of writing. New England: Edinburgh University Press.

Martínez, J. (2015). El género Tesis Doctoral de Historia y Física: Descripción y variación retórico-funcional. In G. Parodi, \& G. Burdiles. (Eds.), Leer y escribir en contextos académicos y profesionales: Géneros, corpus y métodos (pp. 113-152). Santiago de Chile: Ariel.

Massi, M. (2005). Las citas en la comunicación académica escrita. Revista Iberoamericana de Educación, 36(5), 1-7.

McCambridge, L. (2019). If you can defend your own point of view, you're good: Norms of voice construction in student writing on an international Master's programme. English for Specific Purposes, 54, 110-126. https://doi.org/10.1016/j.esp.2019.01.003 
secciones de Tesis de lingüistica: Determinación de la variación entre grados académicos [Tesis de Doctorado, Pontifica Universidad Católica de Valparaíso] Repositorio ANID http://repositorio.conicyt. cl/handle/10533/188574. https://doi.org/10.13140/RG.2.1.1053.0800 OECD. (2019). Education at a glance. https://doi.org/10.1787/889e8641en

Qingrong, L., \& Deng, L. (2019). Chinese MA Student Writers' Identity Construction in Citation Practices. Chinese Journal of Applied Linguistics, 42(3), 365-385. https://doi.org/10.1515/CJAL-2019-0022 Reyes, G. (1993). Los procedimientos de cita. Estilo directo y estilo indirecto. Madrid, España: Arco Libros.

Sabaj, O., \& Paéz, D. (2011). Tipos y funciones de las citas en artículos de investigación de tres disciplinas. Literatura y Lingüistica, (22), 117-134.

Sala-Bubaré, A., \& Castelló, M. (2018). Writing regulation processes in higher education: a review of two decades of empirical research. Reading and Writing, 31(4), 757-777. https://doi.org/10.1007/s11145017-9808-3

Salami, M., \& Olatokun, W. (2018). Citation analysis of Ph.D. theses at faculty of science, university of Ibadan, Nigeria. Issues in Science and Technology Librarianship, (89), 1-9. https://doi.org/10.5072/ FK29Z97H4B

Shi, L., Fazel, I., \& Kowkabi, N. (2018). Paraphrasing to transform knowledge in advanced graduate student writing. English for Specific Purposes, 51, 31-44. https://doi.org/10.1016/j.esp.2018.03.001

Simpson, S. 2016. New frontiers in graduate writing support and program design. In S. Simpson, N. Caplan, M. Cox, \& T. Phillips. (Eds.), Supporting graduate student writers : research, curriculum, and program design (pp. 1-20). EUA: University of Michigan Press.

Solar, I. (2013). Descripción funcional de introducciones de tesis doctorales en las disciplinas de química y lingüística. Onomázein, 28(2), 72-87. https://doi.org/10.7764/onomazein.28.7

Swales, J. (1990). Genre Analysis: English in Academic and Research Settings. Cambridge: Cambridge University Press.

Swart, A. (2019). Citation analysis of master dissertations at the central university of technology, South Africa. African Journal of Library Archives and Information Science, 29(2), 115-129.

UNESCO \& IESLAC. (2007). Informe sobre la Educación Superior en América Latina y el Caribe 2000-2005. Instituto Internacional de la UNESCO para la Educación Superior en América Latina y el Caribe (IESALC). 
Venegas, R, Meza, P., \& Martínez, J. (2013). Procedimientos discursivos en la atribución del conocimiento en tesis de lingüística y filosofía en dos niveles académicos. Revista de Lingüística Teórica y Aplicada, 5l(1), 153-179. https://doi.org/10.4067/S0718-48832013000100008

White, W., \& Grinnell, J. (2011). The Ed.D. v2.0: Imagining a new doctorate in education. In D. Callejo, S. Fain, \& J. Slater. (Eds.), Higher education and human capital: Re/thinking the doctorate in America. EUA: Sense Publishers.

Zavala, V. (2011). La escritura académica y la agencia de los sujetos. Cuadernos Comillas, 1, 52-66.

Recebido em: 15/03/2021

Aprovado em: 26/08/2021 